\title{
Stereological Analysis of Colon Under Exposure of L-Arginine and L-NAME in Female Wistar Rats
}

\author{
Keywan Mortezaee, ${ }^{1}$ Fatemeh Sabbaghziarani, ${ }^{1}$ S. Mohammad Hossein Noori-Mugahi, ${ }^{1,}$ Fatemeh \\ Moghani-Ghoroghi, ${ }^{1}$ and Makan Sadr ${ }^{1}$ \\ ${ }^{1}$ Department of Anatomy, School of Medicine, Tehran University of Medical Sciences, Tehran, IR Iran \\ "Corresponding author: S. Mohammad Hossein Noori-Mugahi, Department of Anatomy, School of Medicine, Tehran University of Medical Sciences, Tehran, IR Iran. E-mail: \\ noorimoo@gmail.com
}

Received 2014 August 27; Accepted 2014 November 12.

\begin{abstract}
Background: Nitric oxide (NO) as a signaling molecule plays a predominant role in different physiological and pathological processes in a variety of organs such as the gastrointestinal (GI) tract. It is produced from L-arginine by nitric oxide synthase (NOS), which in turn is inhibited by L-NG-nitroarginine methyl ester (L-NAME).

Objectives: This study aimed to stereologically analyze colon under exposure of L-arginine and L-NAME in female Wistar rats.

Materials and Methods: In this experimental study, 40 female rats (200 - $250 \mathrm{~g}, 8$ weeks age) were divided into 5 groups ( $\mathrm{N}=8$ ). Normal saline $(2 \mathrm{~mL} / \mathrm{kg})$, L-arginine $(200 \mathrm{mg} / \mathrm{kg})$, L-NAME $(20 \mathrm{mg} / \mathrm{kg})$ and L-arginine + L-NAME (with the same doses) were administered i.p. for 3 days. After 2 weeks, the colon was removed, stained with hematoxylin and eosin ( $\mathrm{H}$ and $\mathrm{E}$ ) and evaluated under light microscopy. Colonic mucosal thickness, gland height and diameter, and total thickness of the wall were measured using optical software and analyzed by one-way ANOVA followed by Tukey's post hoc test using SPSS-16. $\mathrm{P}<0.05$ was considered statistically significant.

Results: The data of the present work revealed relatively narrow ranges of decrease in mean variables compared to the control group, which was not significant.

Conclusions: Despite the remarkable role of NO in GI tract, the results demonstrated no significant effect of L-arginine and L-NAME on colon parameters.
\end{abstract}

Keywords: Nitric Oxide, L-arginine, L-NAME, Colon, Rat

\section{Background}

Nitric oxide (NO) is one of the smallest biosignaling molecules and a free radical [1-3], involved in many physiological and pathological processes due to its important role in mammal cellular signaling [4]. Some sources equate NO and endothelial-derived relaxing factor (EDRF) produced by vascular endothelial cells with a significant impact on smooth muscle relaxation, consequent vasodilatation and increasing the blood flow [3-5].

This function along with platelet aggregation and leukocyte adhesion to the endothelium made NO an essential factor in vascular homeostasis [6]. Two relative processes for biosynthesis of NO are as follows: by NOs acting on L-arginine, oxygen and NADPH and also by nitrate reduction [3-5]. Three isoforms of NOs exist; at the GI system, the two constitutively expressed isoforms i.e. neuronal NOs (nNOs) and endothelial NOs (eNOs) are pointed mainly at the enteric nervous system and vascular endothelium, respectively. NO is generated at many sites in the GI tract. Evidence suggests the colonic epithelium to be the source of NO production, which is highly regulated by cytokines [7]. NO mediates multiple physiological functions in the GI tract and plays a key role in mucus secretion and mucosal integrity, which itself leads to homeostasis maintenance. Undoubtedly, disruption of these beneficial aspects can expose GI tract to pathological conditions [8].

The principal inhibitory role of $\mathrm{NO}$ as a neurotransmitter in controlling GI tract motility is now strongly under consideration for related disorders. Based on animal research, NOs inhibition is associated with colon contraction [9]. L-NAME is inhibitor of NOs, which causes endothelialdependent contraction in vessels and inhibits endothelialdependent relaxation. L-NAME provides a new mechanism for development of hypertension [10].

\section{Objectives}

Based on the mentioned research related to the role of NO in different parts of GI system especially colon, we designed the present study to analyze stereologically colon 
under exposure of L-arginine and L-NAME in female Wistar rats.

\section{Materials and Methods}

This study is an interventional experimental research. Forty female Wistar albino rats weighing 200 - $250 \mathrm{~g}$ with an average age of 8 weeks were obtained from laboratory animal center, faculty of pharmacy at Tehran University of Medical Sciences. In this study, animal care conditions were 12 hour periods of light (07:00 - 19:00) and darkness (19:00 - 07:00), $23 \pm 2^{\circ} \mathrm{C}$ temperature and enough food and water. All animal experiments were carried out according to the guidelines of the Iranian council for use and care of animals and were approved by the animal research ethical committee of Tehran University of Medical Sciences. Then, five rats were kept in each cage under controlled conditions of light, temperature and humidity. Animals were assigned to the following groups: Control, Sham (2 $\mathrm{mL} / \mathrm{kg}$ normal saline), L-arginine (200 mg/kg), L-NAME (20 $\mathrm{mg} / \mathrm{kg}$ ) and combination of L-arginine + L-NAME with the same dose (Sigma, Germany) via i.p. injection at days 3 5 after evaluation. On day 18, rats were anesthetized with ether. After abdominal opening, colon was resected and fixed in $10 \%$ formalin; the colon samples were harvested, processed, and sectioned -by microtome; then, slices 5, 8, 11, 14 , and 17 (5 $\mu$ m thickness) from each section were chosen, stained, and observed under light microscope (Olympus CX31, Japan). Four parameters (mucosal thickness, gland height and diameter, and total thickness) of the colon wall were measured via micrometric criteria by Image tool III software. Statistical analysis was performed by one-way ANOVA followed by Tukey's post hoc test using SPSS-16 (Microsoft, IL, USA) to evaluate the level of significance between different groups. $\mathrm{P}<0.05$ was considered statistically significant.

\section{Results}

Table 1 provides information about the changes in the mean \pm standard deviation (SD) of 4 variables. It is clear from the data that there were relatively narrow ranges of decrease in mean variables in comparison to the control group, which were not significant.

The lowest alterations in mucosal thickness and gland height and diameter were seen in L-arginine + L-NAME group with 498.39, 486.05, and $95.04 \mu \mathrm{m}$ respectively, whereas this rate was higher for total thickness of the wall. The greatest changes in mucosal thickness and gland height were seen in L-arginine and L-NAME groups, respectively with 462.98 and $439.69 \mu \mathrm{m}$ values. Figure $1 \mathrm{~A}$ - E and
Figure 2A - D also indicate colon wall characteristics in different groups.

\section{Discussion}

In this study the effect of L-arginine and L-NAME on colon characteristics was evaluated. The data showed that there were no significant changes in mean variables including mucosal thickness, gland height and diameter, and total wall thickness in comparison to the control group.

The results showed that there were no significant changes in L-arginine group in comparison with control group which was consistent with Puiman et al. results. They reported that intestinal infusion of arginine caused only a slight increase in intestinal mucosal growth and there was no significant change with regard to protein synthesis or cell proliferation [11]. Based on the conditions under which this process takes place, reaction of NO with other radicals can have two different consequences: one beneficial and one deleterious [8]. Arginine is an essential precursor for poly amines and NO, which are involved in many different processes namely gut mucosal repair, enhancing cell migration, increasing the blood flow, cell number and height augmentation, and activation of protein synthesis. Low arginine content decreases NO synthesis and subsequently the blood flow in the small intestine of rat $[11,12]$. Whereas another research has demonstrated the function of NO in high levels, which is induced by iNOs, may cause damage to the intestinal epithelial cells [13]. It should be mentioned that there is no cytotoxic effect of NO, by itself, on intestinal tissue; on the other hand, eNOs derived NO regulate crucial functions of the GI mucosa, such as epithelial and microvascular permeability. The former markedly reflects the functional integrity of the GI mucosa barrier, which its disruption assumed as a quantitive index for colonic injuries. Decrease in epithelial permeability exerts through NO is regard to its stimulatory effects on the cGMP content of intestinal epithelia and inhibitory effects on secretion of histamine and platelet-activation factor (PAF) by mucosal mast cells [14]. Furthermore, NO activates gastric guanylate cyclase and stimulates colonic electrolyte secretion through cholinergic receptors which in turn mediated by prostaglandin E2 (PGE2) $[15,16]$.

Animal and human experimental and clinical studies have reported different data of NO effects on GI tract. In rat model, administration of NO into the gastric lumen led to a dose-dependent increase in mucus gel thickness. Proven evidence shows another beneficial effect of exogenous NO by protecting rat gastric mucosa form some toxic injuries; perhaps this function is related to the ability of NO in 
Table 1. Comparison of Four Variables Among the Study Groups ${ }^{\mathrm{a}}$

\begin{tabular}{|c|c|c|c|c|c|}
\hline Study Groups & Mucosal Thickness & Gland Height & Gland Diameter & Total Thickness & Significanc \\
\hline Control & $538.89 \pm 30.63$ & $490.56 \pm 29.57$ & $105.98 \pm 10.11$ & $661.53 \pm 58.19$ & NS \\
\hline Normal saline & $492.8 \pm 52.13$ & $452.75 \pm 57.35$ & $82.48 \pm 1.29$ & $621.97 \pm 25.54$ & NS \\
\hline L-arginine & $462.98 \pm 60.26$ & $474.98 \pm 81.49$ & $88.63 \pm 7.43$ & $652.58 \pm 31.91$ & NS \\
\hline L-NAME & $484.52 \pm 49.06$ & $439.69 \pm 46.70$ & $92.03 \pm 12.90$ & $652.62 \pm 31.75$ & NS \\
\hline L-arginine + L-NAME & $498.39 \pm 31$ & $486.05 \pm 55.29$ & $95.04 \pm 19.29$ & $693.92 \pm 21.10$ & NS \\
\hline
\end{tabular}

${ }^{\mathrm{a}}$ Values are expressed as mean $\pm \mathrm{SD}$ and the Variables unit is $\mu \mathrm{m}$.

NS; Non-significan

maintaining mucosal blood flow and inhibiting leukocyteendothelial cell interactions [8]. In humans, NOS inhibition did not change rectal motor or sensory response to distension, while it increased the motility of the distal ileum and proximal colon [9]. Although markedly increased NO synthesis has been shown by scientists in some types of colonic disorders (for instance, in irritable bowel syndrome), the cellular source of $\mathrm{NO}$ in the mucosa is still unknown [7].

Other reports reveal that, L-NAME reduces cell proliferation and it can act as a protective factor in colon cancer due to chemical agents [17]. Furthermore it induces a dose-dependent increase in systemic blood pressure and a decrease in resting gastric mucosal blood flow which causes leaker mucosal barrier through cGMP diminish [8, 14]. These reports are consistent with our results. In the current experiment, L-NAME had a roughly slight decreasing impact on variables compared to the control group, but these changes were not significant.

Also a similar result was observed in L-arginine + LNAME group, which can be due to cessation of intestinal absorption or increase breakdown of L-arginine by L-NAME [11]. Some scientists believe that exogenous L-arginine can prevent the effects of NO synthesis inhibitors on epithelial regeneration [18] which was inconsistent with our results.

In conclusion, our data indicated that L-arginine and L-NAME had no significant effect on the understudy colon characteristics. Furthermore, evaluation of apoptosis markers and different doses of NO precursor and inhibitor is suggested.

\section{Acknowledgments}

The authors would like to express their gratitude to the anatomy department and histotechnique laboratory of Tehran University of Medical Sciences for their endless support in conduction of this study. This article is not the result of a thesis or a research proposal.

\section{Footnotes}

Authors' Contribution: All authors had equal role in design, work, statistical analysis, and manuscript writing.

Financial Disclosure: The authors declare no conflict of interest.

Funding/Support: Tehran University of Medical Sciences. 
Figure 1. Transverse Sections of Colon in Different Groups
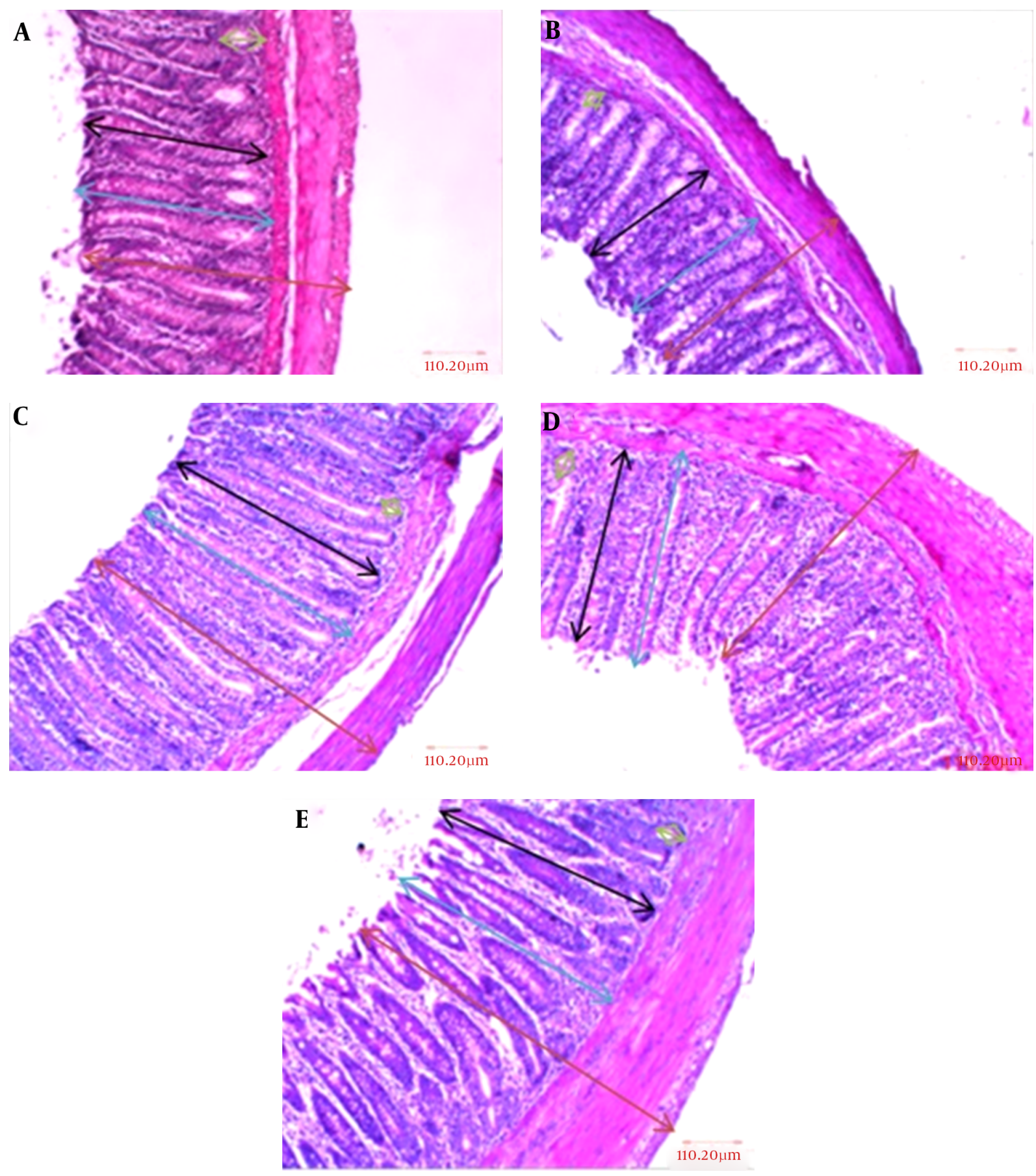

A, Control; B, normal saline; C, L-arginine; D, L-NAME and E, L-arginine + L-NAME (H \& E, $100 \times$ ). As it could be seen, there were no significant changes in mucosal thickness, gland height and diameter, and total thickness; the arrows for the parameters were blue, black, green, and brown respectively. 
Figure 2. The Mean Value of Different Variables

A

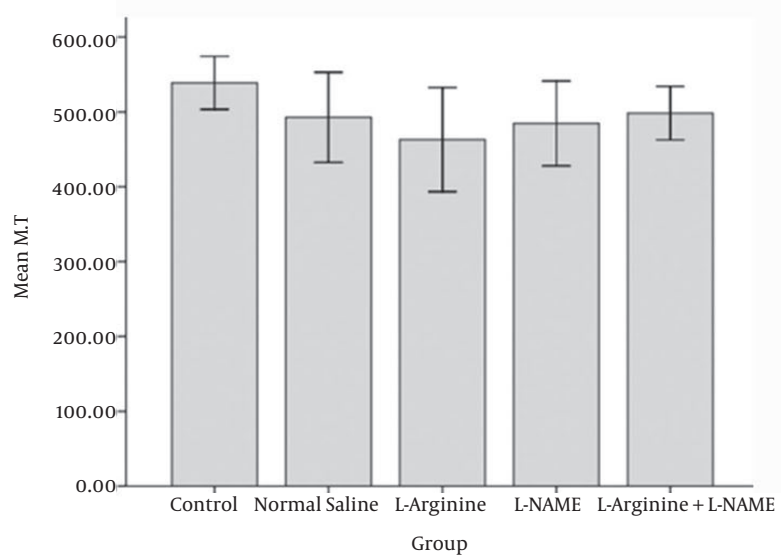

C

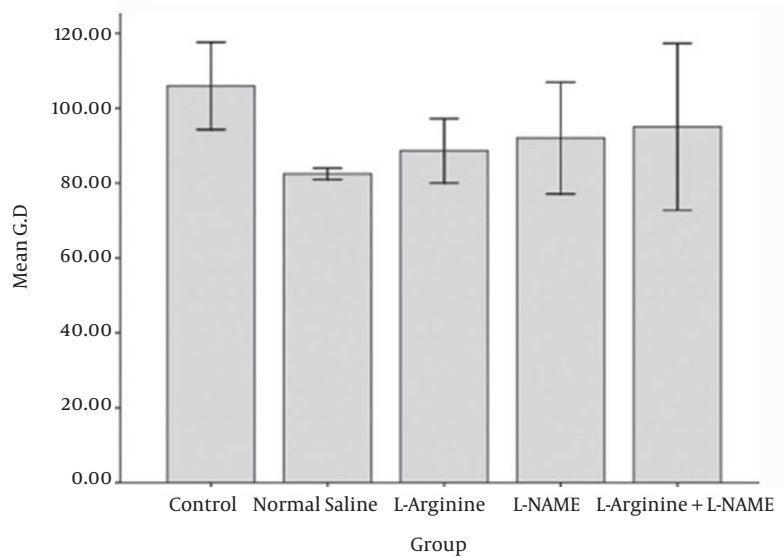

B

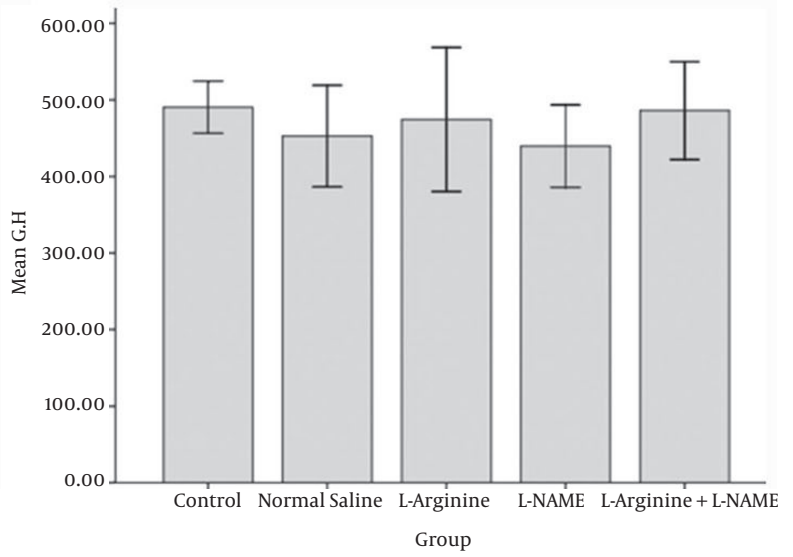

D

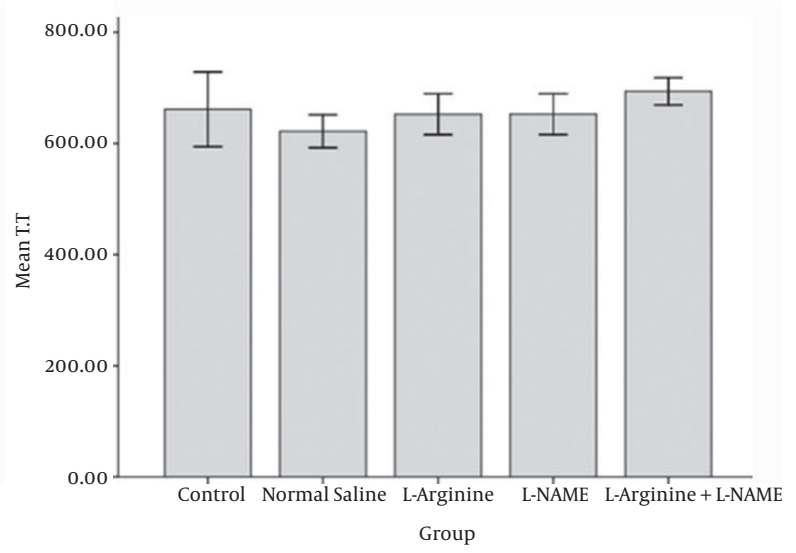




\section{References}

1. Derakhshan B, Hao G, Gross SS. Balancing reactivity against selectivity: the evolution of protein S-nitrosylation as an effector of cell signaling by nitric oxide. Cardiovasc Res. 2007;75(2):210-9. doi: 10.1016/j.cardiores.2007.04.023. [PubMed: 17524376].

2. Kyro C, Skeie G, Dragsted LO, Christensen J, Overvad K, Hallmans G, et al. Intake of whole grain in Scandinavia: intake, sources and compliance with new national recommendations. Scand J Public Health. 2012;40(1):76-84. doi: 10.1177/1403494811421057. [PubMed: 21976053].

3. Tazawa H, Kawaguchi T, Kobayashi T, Kuramitsu Y, Wada S, Satomi Y, et al. Chronic inflammation-derived nitric oxide causes conversion of human colonic adenoma cells into adenocarcinoma cells. Exp Cell Res. 2013;319(18):2835-44. doi: 10.1016/j.yexcr.2013.08.006. [PubMed: 23948305].

4. Hou YC, Janczuk A, Wang PG. Current trends in the development of nitric oxide donors. Curr Pharm Des. 1999;5(6):417-41. [PubMed: 10390607].

5. Akintunde O, Adenowo TK, Kehinde B. Some adverse effects of nitrite on oxidative status and histological structures of adult male wistar rats testes. Ame J Res Commun. 2014;2(9):227-41.

6. Dessy C, Feron O. Pathophysiological roles of nitric oxide: in the heart and the coronary vasculature. Curr Med Chem Antiinflamm Antiallergy Agents. 2004;3(3):207-16.

7. Kolios G, Rooney N, Murphy CT, Robertson DA, Westwick J. Expression of inducible nitric oxide synthase activity in human colon epithelial cells: modulation by T lymphocyte derived cytokines. Gut. 1998;43(1):56-63. [PubMed: 9771406].

8. Lanas A. Role of nitric oxide in the gastrointestinal tract. Arthritis Res Ther. 2008;10 Suppl 2:S4. doi: 10.1186/ar2465. [PubMed: 19007429].

9. Corsetti M, Vos R, Gevers A, Demedts I, Janssens J, Tack J. Influence of nitric oxide synthase inhibition on the motility and sensitivity of distal colon in man. Neurogastroenterol Motil. 2013;25(4):e256-62. doi: 10.1111/nmo.12093. [PubMed: 23510091].
10. Luscher TF, Vanhoutte PM. The endothelium: Modulator of cardiovascular function. Boston: CRC press; 1990. p. 248.

11. Puiman PJ, Stoll B, van Goudoever JB, Burrin DG. Enteral arginine does not increase superior mesenteric arterial blood flow but induces mucosal growth in neonatal pigs. J Nutr. 2011;141(1):63-70. doi: 10.3945/jn.110.131888. [PubMed: 21106927].

12. Noori-Mugahi SMH, Moghani-Ghoroghi F. Morphometry of Rat Jejunal Enterocytes Number and Height after L-Arginine and L-NAME Administration. Zahedan J Res Med Sci. 2015;17(2):27-30.

13. Lamarque D, Kiss J, Tankovic J, Flejou JF, Delchier JC, Whittle BJ. In duction of nitric oxide synthase in vivo and cell injury in rat duodenal epithelium by a water soluble extract of Helicobacter pylori. $\mathrm{Br}$ J Pharmacol. 1998;123(6):1073-8. doi: 10.1038/sj.bjp.0701706. [PubMed: 9559888].

14. Kolios G, Valatas V, Ward SG. Nitric oxide in inflammatory bowel disease: a universal messenger in an unsolved puzzle. Immunology. 2004;113(4):427-37. doi: 10.1111/j.1365-2567.2004.01984.x. [PubMed: 15554920].

15. Brown JF, Keates AC, Hanson PJ, Whittle BJ. Nitric oxide generators and cGMP stimulate mucus secretion by rat gastric mucosal cells. Am J Physiol. 1993;265(3 Pt 1):G418-22. [PubMed: 8214062].

16. Wilson KT, Vaandrager AB, De Vente J, Musch MW, De Jonge HR, Chang EB. Production and localization of CGMP and PGE2 in nitroprussidestimulated rat colonic ion transport. Am J Physiol. 1996;270(3 Pt 1):C832-40. [PubMed: 8638664].

17. Akguen-Dar K, Balci H, YADCI A, Kapucu A, Uyaner I. Effects of leptin on the epithelial cell proliferation from the small intestine and nitric oxide (NO) production in rats [in France ]. Rev Med Vet. 2007;158(3):161-8.

18. Gookin JL, Rhoads JM, Argenzio RA. Inducible nitric oxide synthase mediates early epithelial repair of porcine ileum. Am J Physiol Gastrointest Liver Physiol. 2002;283(1):G157-68. doi: 10.1152/ajpgi.00005.2001. [PubMed: 12065303]. 